\title{
Systematic review protocol: following PRISMA guide from Cochrane to generate evidence as treatment effect of pharmaceutical care for hypertension in primary care
}

\author{
Maurílio de Souza Cazarim ${ }^{\oplus 1,5 *}$; Tiago Marques dos Reis $^{2}$; \\ Estael Luzia Coelho da Cruz-Cazarim³ ${ }^{3}$ Leonardo Régis Leira Pereira ${ }^{4}$
}

${ }^{1}$ PhD. Pharmaceutical Sciences department, Pharmaceutical Services and Clinical Pharmacy Research Center (CPAFF), School of Pharmaceutical Sciences of Ribeirão Preto, University of São Paulo, Ribeirão Preto, São Paulo, Brazil. ${ }^{2}$ PhD. Professor of Faculty of Pharmaceutical Sciences, Federal University of Alfenas, Alfenas, Minas Gerais, Brazil. ${ }^{3}$ MSc. Pharmacist of Pharmaceutical Service of School of Pharmaceutical Sciences of Ribeirão Preto, University of São Paulo, Ribeirão Preto, São Paulo, Brazil. ${ }^{4}$ Professor of Department of Pharmaceutical Sciences department, Pharmaceutical Services and Clinical Pharmacy Research Center (CPAFF), School of Pharmaceutical Sciences of Ribeirão Preto, University of São Paulo, Ribeirão Preto, São Paulo, Brazil. ${ }^{5}$ Professor of Faculty of

Farmacy of Federal University of Juiz de Fora, Juiz de Fora, Minas Gerais, Brazil

\begin{abstract}
Pharmaceutical care (PC) is in the implementation process in Brazil and Latin America. Synthesis of evidence has been requested for monitoring and evaluating the process regarding the treatment effect. The objective is to build and disseminate a systematic review protocol to make a standard for updating results from pharmaceutical care for hypertension and for other diseases. This is a protocol for systematic review studies regarding a real example of a protocol reasoned in pharmaceutical care for hypertension in primary care. This protocol was delineated grounded in the Cochrane Handbook. Descriptors and words were defined using MeSH (Medical Subject Headings), DeCS (Descriptors in Health Sciences) and Emtree thesaurus, and the search was performed in English, Spanish and Portuguese, without filters, up to March, 27th, 2017. The results were structured in the PRISMA flowchart. Results found from all databases were: the Cochrane Library $(n=202)$; PubMed $(n=2608)$; LILACs $(n=909)$; Embase $(n=$ 1653); Scopus ( $n=1298)$; IPA ( $n=967)$; and Web of Sciences $(n=435)$. From these, 1688 were duplicate articles. The content of this paper can aid the constant monitoring of pharmaceutical care implementation and contribute to the improvement of the quality and evidence levels of published studies.
\end{abstract}

Keywords: Pharmaceutical services. Hypertension. Primary health care. Review literature as topic. Evidence-based medicine.

\section{INTRODUCTION}

According to the World Health Organization WHO (2017), systemic arterial hypertension (SAH) is

\footnotetext{
*Correspondence: M. S. Cazarim. Centro de Pesquisa em Assistência Farmacêutica e Farmácia Clínica (CPAFF). Faculdade de Ciências Farmacêuticas de Ribeirão Preto. Universidade de São Paulo. Sala 23, bloco S. Av. Café, s/no 14040-903, Ribeirão Preto - SP, Brasil. Phone: +55 16 3315-4236. E-mail: maurilio.jf@gmail.com
}

a chronic disease considered to be the main risk factor for cardiovascular disease (CVD). In 2008, 17.3 million people died due to CVD, with the highest prevalence of coronary diseases and stroke, with 7.3 and 6.2 million deaths respectively. In 2012, the global figure reached 17.5 million deaths, with the estimate for 2030 being 23.3 million deaths from CVD. It is estimated that 9.4 million deaths from CVD in 2010 had SAH as an associated factor, or $16.5 \%$ of global deaths (WHO, 
2013; WHO, 2014). In Brazil, hypertension affects approximately one third of the population, reaching more than $60 \%$ in the elderly and $5 \%$ of the 70 million children and adolescents (Malachias et al., 2016). In addition, approximately 1.2 million hospitalizations are linked to CVD, and approximately 4.5 hospitalizations per 1,000 inhabitants are for $\mathrm{SAH}$, generating an approximate cost of US\$ 650 million per year (BSC, 2010; Malachias et al., 2016).

An alternative considered as a health technology capable of providing better perspectives in the control of chronic diseases is Pharmaceutical Care (Zubioli et al., 2013; Pereira \& Freitas, 2008; Lyra, Marcellini, Pelá, 2008; Castro \& Correr, 2007; Araújo, Ueta, Freitas, 2005). Pharmaceutical care is the philosophy of professional practice that requires a clinical and humanistic training of pharmacists to ensure the success of pharmacotherapy. This process involves activities such as consultations, interventions, records, and individualized follow-up of medication treatment, following a well-defined therapeutic plan until the patient is discharged (OPAS, 2002).

Regarding this health technology, the pharmacist is responsible for making a screening over health conditions and pharmacotherapy of the patient. By identifying the incorrect use of medicines or need of investigation about the patient's pharmacotherapy to improve outcomes and quality of life, the pharmacist is able to invite the patient for a follow-up, conducting periodic consultations. The initial consultation consisted of the collection of socio-demographic data, clinical history and life habits. Subsequent consultations are needed for monitoring indicators such as: blood pressure measurements and cardiovascular risk measures; and actions such as: analysis of medications and test results, education in health matters with guidelines on patient behavior regarding life habits, adherence to treatment and, when necessary, interventions in pharmacotherapy up to patient discharge (Cipolle, Strand and Morley, 1998; Pereira \& Freitas, 2008).

Pharmaceutical care has been shown to be effective in the control of SAH and the reduction of
CVD risk (Aguwa, Ukwe, Ekwunife, 2008; Lyra, Marcellini, Pelá, 2008). As evidenced by Souza et al. (2007), pharmaceutical care was able to reduce systemic blood pressure by almost $20 \mathrm{mmHg}$ and 10 $\mathrm{mmHg}$ for diastolic blood pressure in hypertensive patients. Consequently, the care of hypertensive patients promoted by pharmaceutical care has been important in reducing cardiovascular risk. However, the literature has not shown a consensus regarding the impact of different models of pharmaceutical care on cardiovascular risk factors such as blood pressure and cholesterol levels in patients with SAH.

Consolidating a systematic review protocol regarding this issue would be useful for updating results from pharmaceutical care of different diseases, not only hypertension, in primary care. It is noteworthy that the Brazilian Ministry of Health has initiated pharmaceutical care implementation in the Public Health System. There are incentive strategies in the country as well as a project by the Institutional Development Program of the Public Health System for training and qualification of pharmacists to work developing pharmaceutical care in primary care (Brazil, 2018). Pharmaceutical care has been a disseminate health technology in America and some developing countries (Brazil, 2012; Brazil 2014).

In this context, the objective of this study was building and dissemination of a systematic review protocol to make a standard for updating results from pharmaceutical care for hypertension and, with some adaptations, for other diseases.

\section{METHODS}

This is a protocol for elaboration of a systematic review regarding the results of pharmaceutical care for hypertension in primary care. This systematic review protocol presumed a structure according to the Cochrane guide for systematic review and meta-analysis (Higgins, Green, 2011). A flowchart was built for systematizing a structure for a systematic review that contemplates not only clinical trials (Figure 1). 


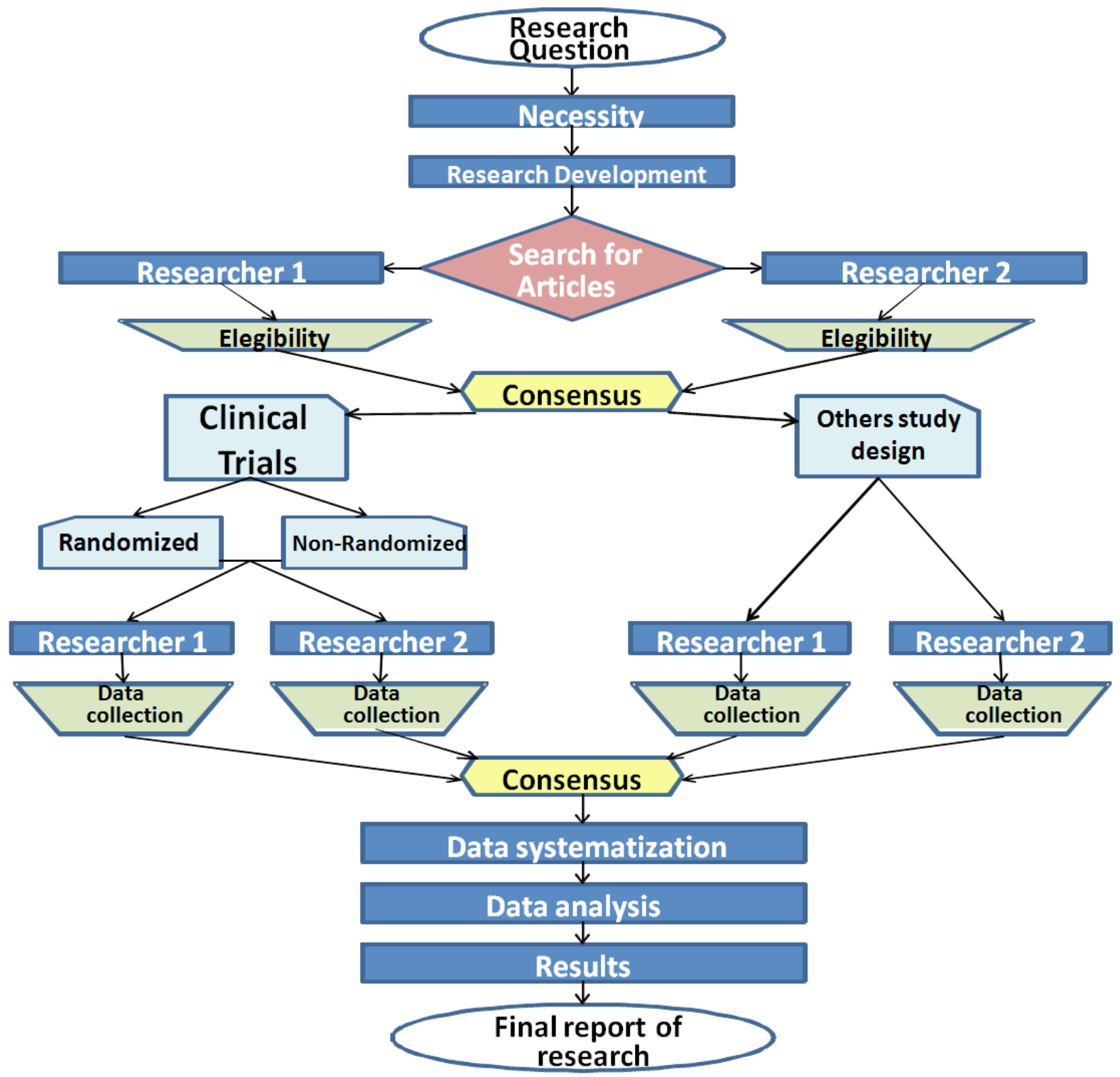

FIGURE 1 - Systematic review flowchart.

\section{Register}

This study protocol was registered in the PROSPERO databases, which gives it validation and allows researchers and readers to follow the systematic review, as well as access information about and maintaining contact with the researchers responsible. The page can be accessed at $<$ https://www.crd.york. ac.uk/prospero/ $>$ and the registration number of this protocol is: CRD42017079761, titled "Clinical outcomes achieved by pharmaceutical care for patients diagnosed with systemic arterial hypertension under primary care".

\section{Review question}

What pharmaceutical care models for pharmacotherapeutic monitoring have been developed for hypertensive patients in primary care and what is their clinical impact?

\section{Aim of the systematic review grounded in this protocol}

The aim of a systematic review linked to this protocol should be to know the different models of practice of pharmaceutical care for hypertension in primary care and to summarize the clinical results achieved by this health technology. 
Search

Cochrane advises the use of a minimum of three important databases recognized as benchmarks for developing a Systematic Review regarding Health based in Evidence, such as: Cochrane, PubMed, Scopus or EMBASE and, LILACS for searches that need to include studies from Latin America. It is always important to define the time of the search for all databases, because the search in a systematic review is not punctual, and the researchers can spend more than one day retrieving studies in databases.

\section{LILACS, PUBMED, COCHRANE Databases}

LILACS, PubMed and Cochrane are referential databases (they record the summary study data and lead the researcher to the journal editor). Periodicals are database-related, such as Medline for the health area. It is noted that in MEDLINE are categories of journals that group editorial groups such as Elsevier and databases of periodicals such as Science direct, and it is noteworthy that Elsevier is an editorial group, just as Cochrane has the Wiley online library. It is important to know that in the search, when we place the word(s) between parentheses it means to be in the same text; between quotation indicates it is to find exactly the form that is written; to search by words must be without quotation marks and parentheses; when keywords are used they should be considered language, but when descriptors are used this is not necessary; the descriptors retrieve the article in the database, and it is not necessarily related to the content.

Articles are indexed to a subject and because of this it is important to also search for keywords. It should be emphasized that articles indexed by subjects are different to periodicals indexed to a database, since the latter need to contemplate other criteria to achieve indexation, such as publication indexes and citations. Another important fact is the difference between the databases for retrieval of documents, for example, in the LILACS database, when the " $\$$ " symbol is placed it extends to searches by suffixes; in other databases the "*" symbol is usually for the same. For Boolean operators, the search groups must be divided according to PICO and PECO, the combination must be made among groups by the operator "AND" and intra-groups by "OR", in which the terms must always be between parentheses, because unlinking the "OR" in the search can find results outside the target and provide a broad imprecise research.
In LILACS one must perform the search in the Portuguese, English and Spanish languages. The search strategy performed can use only the word in English such as the descriptor, but it is recommendable to use the same word in its DeCS translation for Spanish and Portuguese because the search occurs in other fields and the database recognizes documents in those languages. Compound words should be performed as follows: "first word AND second word", because LILACS does not recognize the compound word in fields such as abstracts and titles, it only recognizes in the field descriptor.

In the search strategy for PubMed, when the search by descriptor is put as follows: "word" [Mesh], it means that only this term will be searched, and according to the $\mathrm{MeSH}$ hierarchy, the words that are assigned in the term do not enter; if you add the word "terms" as [Mesh terms], synonymous related to the descriptor are also searched for. It is important not to mark the box "do not explode" because if it is not checked it will explode the search for the hierarchy of descriptors. It is worth noting that whenever the phrase "not quoted" appears for any word in the search, it is necessary to revise the word because the database will make an expanded retrieval of documents related to variations of possible words, which will be associated with that "wrong" word, and the search result may then be overestimated as to retrieval of documents, and the result of the search can be overestimated.

In the Cochrane library the same strategy of PubMed can be used, but the database is different for recognizing the documents. Typing the descriptor by descriptor in each line is needed and, the same should be made for words. After this, the search should be performed by combining each line for "PICO" using the intra combinations "OR" and "AND" between categories $\mathrm{P}, \mathrm{I}, \mathrm{C}, \mathrm{O}$; the combination will be by hash tag and the number of line, e.g.: \#1; \#2 etc.

\section{EMBASE Databases}

In EMBASE the search always seeks for descriptor and word in the same strategy. Descriptors and exploded search will be found in the dictionary "Browse Emtree". The search is expanded when the term in "Find term" is selected and is thus assigned to explode, or the adjacent terms are selected. It does the same as PubMed, if the search is performed by word it searches more broadly than descriptors. Multidisciplinary databases such as SCOPUS cannot expand the search by descriptors, the search is performed only by word. 


\section{SCOPUS and Web of Sciences Databases}

In a search carried out in these databases, it is advisable not to search in all fields, because the wide scan will be performed, even in the references. Ideally there is no loss of sensitivity for the search in specific fields when occurring by title, abstract and keywords. These databases search for words and do not have a controlled vocabulary, thus the search will always be by keyword. It is important to make sure that the compound words are enclosed in quotation marks. It is possible to perform the search according to PubMed, but without the word "Mesh", because these are multidisciplinary databases and do not have descriptors. The "\$" symbols replace one or no letter, in this last case it only closes the word; "?" Replaces one letter; "*” Replaces the suffix, and is not useful for prefixes. For a good sensitivity it is important to use the descriptors found in EMBASE as the search words in these databases. Note that you do not need to switch to other languages.

Web of Sciences is the same strategy of PubMed, but there is no "Mesh" to select and "Topic", and when using words, it should preferably be selected to be found in all fields. The search should be performed by category in "quick search", first the P of PICO, enter the descriptors and select topic; put the words and select all fields, then click Search. This search will be saved in your history; go back and repeat for the letter I; and consequently to $\mathrm{C}$ and $\mathrm{O}$. Then access the history and select each of the four strategies and click on "Combine/ Match" with AND for the database to show the search results.

SCOPUS is a database that does not accept a lot of words in the search strategy and in the advanced search it is not possible to change the filter to a title. The advanced search should then be performed by typing the filter of "title, abstract and Keyword", e.g. "tit, Abst $<\mathrm{Kw}$ (word) OR (word) ...)". It is necessary to be careful when mixing expressions from one database with another since "no quoted phrase" will appear in the search, and when this occurs the database searches in an exploratory way, dismembering the word.

\section{Search for free articles and Gray literature}

It is recommended when making a search for a Digital Object Identifier System (DOI) when the article is known and you intend it to be analyzed, the access can be: $<$ dx.doi.org $>$. Other databases specific for any country or Institution can be accessed to improve the sensitivity of the search. It is important to perform the search for non-indexed articles and abstracts or proofs that can be important for the study. It is recommended to perform a search of the Gray literature, which can be in local specific databases or web navigators such as Google ${ }^{\circledR}$.

\section{Search design}

The search strategies were designed for the following databases: MEDLINE ${ }^{\circledR}$ (March 27, 2017), EMBASE (March 27, 2017), Scopus (March 27, 2017), LILACs (March 27, 2017), CENTRAL Cochrane Library (March 27, 2017), and Web of Science (March 27, 2017). The elaboration of the search had as a principle the strategy outlined by the Cochrane guideline, considering population, intervention/exposure, comparator and in some cases the outcomes (clinical results). However, this review has considered: population, intervention/ exposure, comparator (Higgins, Green, 2011).

\section{Study condition or domain}

Pharmaceutical care developed in primary, preventive or community care should be performed for patients with non-secondary systemic arterial hypertension, and patients should be treated in the primary care setting. Additionally, it should be judged whether the pharmacist performed at least two consultations in a follow-up of the patients of at least three months and also if the results presented by the study came exclusively from the intervention of the pharmacists, themselves being incorporated or not in a multi-professional team (Cipolle, Strand, Morley 1998; Cazarim et al., 2016).

\section{Definition of search categories}

The search was defined in four main categories for clinical trials (PICO) and observational studies (PECO) according to Higgins and Green (2011) (Table I).

The Boolean operators used were "OR" intracategories for word combinations and "AND" intercategories for the combination of the same. It should be noted that three categories were considered: population, intervention/exposure and comparison. No search filters were used. Descriptors and words were extracted from the three main controlled vocabularies 
according to Cochrane, Medical Subject Headings (MeSH) for the Medline database and others, Emtree thesaurus for the EMBASE database, and Health Sciences Descriptors (DeCS) for Latin American databases. It is advisable to make the search strategy with descriptors exploded such as words in three languages from DeCS in the LILACS database. It should be emphasized that the search strategies were adequate for each search database according to its peculiarities (Table II) (Higgins, Green, 2011).
For increasing the sensitivity of the search, entry terms and synonyms for the search strategy in all databases were used, except LILACs, which was specific for Latin America and this was not needed (Table III).

Cochrane emphasizes that the main strategy to be shown in a Systematic review is the PubMed search strategy (Supplemental appendix S1).

TABLE I - Cochrane's acronym method to structure a systematic review

\section{PICO}

\section{PECO}

Population: patients with systemic arterial hypertension

Intervention: pharmaceutical care

Comparator: conventional care of the hypertensive patient in the health system basic care service (all the service offered by the health system in this area without pharmaceutical care)

Outcomes: are not indicated by Cochrane in the strategy because they limit the search too much and lose the search sensitivity.
Population: patients with systemic arterial hypertension

Exposure: non-exposed individuals are those who were assisted by pharmaceutical care, and exposed are those who received only conventional care from the health care system.

Comparator: conventional care of the hypertensive patient in the health system basic care service.

Outcomes: are not indicated by Cochrane in the strategy because they limit the search too much and lose the search sensitivity. 
TABLE II - Search strategy by MeSH, Emtree thesaurus and DeCS linked database

$\begin{array}{lll}\text { MeSH Emtree thesaurus } & \text { DeCS }\end{array}$

Population:

"hypertension" $[\mathrm{MeSH}$

Terms] OR "Blood

Pressure" [MeSH

Terms] OR "Arterial

Pressure"[MeSH Terms]

\section{Intervention:}

"Pharmacy"[MeSH Terms]

OR "Pharmacists" [MeSH

Terms] OR "Pharmaceutical

Services"[MeSH Terms]

Intervention: "pharmaceutical care"/exp OR "clinical pharmacy"/ exp OR "pharmacist"/exp

Comparator: "Primary

Health Care"[MeSH

Terms] OR "Community

Health Services" [MeSH

Terms] OR "Preventive

Medicine" $[\mathrm{MeSH}$

Terms] OR "Secondary

Care"[MeSH Terms]

Population: "hypertension"/ exp OR "blood pressure"/exp OR "arterial pressure"/exp OR "pulse pressure"/exp OR "diastolic blood pressure"/exp OR "systolic blood pressure"/exp

Comparator: "Primary Health Care"/exp OR "Community Care"/exp OR "Preventive Medicine"/exp OR "Secondary Health Care"/exp
Intervention: (Pharmaceutical Care) OR (Community Pharmacy Services) OR (Pharmaceutical Services) OR (Pharmacists) OR (Pharmacy) OR (Pharmacy Residence) OR (Medication Therapy Management) OR (Drug Therapy Management) OR (Pharmacist Intervention)
Population: (Hypertension) OR (Prehypertension) OR (Blood Pressure) OR (Arterial Pressure) OR (Pulse) OR (Blood Pressure High) OR (Diastolic Pressure) OR (Pressure Systolic)
Comparator: (Primary Health Care) OR (Primary Care) OR (Community Health Services) OR (Community Health Care) OR (Preventive Medicine) OR (Comprehensive Health Care) OR (Secondary Care) OR (Secondary Health Care) 
TABLE III - Used words in the search strategy (Entry terms and synonyms)

\section{Population}

Intervention

\section{Comparator}

(hypertension*) OR ("Blood Pressure High") OR ("Blood Pressures High") OR ("High Blood Pressure") OR ("High Blood Pressures") OR ("Pressure Blood") OR ("Blood Pressure") OR ("Pressures Blood") OR ("Blood Pressures") OR ("Diastolic Pressure") OR ("Pressure Diastolic") OR ("Diastolic Pressures") OR ("Pressures Diastolic") OR ("Pulse Pressure") OR ("Pressure Pulse") OR ("Systolic Pressure") OR ("Pulse Pressures") OR ("Pressures Pulse") OR ("Systolic Pressures") OR ("Pressure Systolic") OR ("Pressures Systolic") OR ("Arterial Pressure") OR ("Arterial Pressures") OR ("Pressure Arterial") OR ("Pressures Arterial") OR ("Arterial Tension") OR ("Arterial Tensions") OR ("Tension Arterial") OR ("Tensions Arterial") OR ("Blood Pressure Arterial") OR ("Arterial Blood Pressure") OR ("Arterial Blood Pressures") OR ("Blood Pressures Arterial") OR ("Pressure Arterial Blood") OR ("Pressures Arterial Blood") OR ("Aortic Pulse Pressure") OR ("Aortic Pulse Pressures") OR ("Pressure Aortic Pulse") OR ("Pressures Aortic Pulse") OR ("Pulse Pressure Aortic") OR ("Pulse Pressures Aortic") OR ("Mean Arterial Pressure") OR ("Arterial Pressure Mean") OR ("Arterial Pressures Mean") OR ("Mean Arterial Pressures") OR ("Pressure Mean Arterial") OR ("Pressures Mean Arterial") OR ("Aortic Pressure") OR ("Aortic Pressures") OR ("Pressure Aortic") OR ("Pressures Aortic") OR ("Aortic Tension") OR ("Aortic Tensions") OR ("Tension Aortic") OR ("Tensions Aortic") OR ("Blood Pressure Aortic") OR ("Aortic Blood Pressure") OR ("Aortic Blood Pressures") OR ("Blood Pressures Aortic") OR ("Pressure Aortic Blood") OR ("Pressures Aortic Blood") OR ("Mean Aortic Pressure") OR ("Aortic Pressure Mean") OR ("Aortic Pressures Mean") OR ("Mean Aortic Pressures") OR ("Pressure Mean Aortic") OR ("Pressures Mean Aortic") OR (hypertensive*) OR ("hypertensive patients") OR ("hypertensive patient") OR ("mean artery pressure") OR ("mean blood pressure") OR ("mean arterial blood pressure") OR ("mean artery pressure") OR ("preexistent hypertension") OR ("hypertensive effect") OR ("hypertensive response") OR ("cardiovascular hypertension") OR ("blood tension") OR ("blood tensions") OR ("vascular pressure") OR ("vascular pressures") OR ("arterial pulse pressure") OR ("arterial pulse pressures") OR ("artery pulse pressure") OR ("artery pulse pressures") OR ("pulse tension") OR ("pulse tensions") OR ("artery blood pressure") OR ("artery blood pressures") OR ("artery pressure") OR ("artery pressures") OR ("aorta blood pressure") OR ("blood pressure aorta") OR ("pressure aorta") OR ("aorta blood pressures") OR ("blood pressures aorta") OR ("systemic arterial pressure") OR ("systemic artery pressure") OR ("diastolic blood pressure") OR ("Systolic blood pressure") OR ("blood pressure systolic") OR ("blood systolic pressure") OR ("blood diastolic pressure") OR ("blood pressure diastolic") OR ("systemic arterial pressures") OR ("systemic artery pressures") OR ("diastolic blood pressures") OR ("Systolic blood pressures") OR ("blood pressures systolic") OR ("blood systolic pressures") OR ("blood diastolic pressures") OR ("blood pressures diastolic")
("Medication Therapy Management") OR

("Management Medication Therapy") OR ("Therapy Management Medication") OR ("Drug Therapy Management") OR ("Management Drug Therapy") OR ("Therapy Management Drug") OR ("Pharmaceutical Care") OR ("Care Pharmaceutical") OR ("Pharmacist Interventions") OR ("Pharmacist Intervention") OR ("Pharmacists Interventions") OR ("Pharmacists Intervention") OR ("medication management") OR ("medication reconciliation") OR ("Pharmaceutic Intervention") OR ("Pharmaceutic Interventions") OR ("Pharmaceutical Interventions") OR ("Pharmaceutical Intervention") OR ("Pharmaceuticals Intervention") OR ("Pharmaceuticals Interventions") OR ("Clinical Pharmacists") OR ("Clinical Pharmacist") OR ("Pharmacist Clinical") OR ("Pharmacists Clinical") OR ("Pharmaceutical Service Community") OR ("Pharmaceutical Services Community") OR ("Service Community Pharmaceutical") OR ("Services Community Pharmaceutical") OR ("Pharmacy Services Community") OR ("Community Pharmacy Service") OR ("Pharmacy Service Community") OR ("Services Community Pharmaceutic") OR ("Services Community Pharmacy") OR ("Community Pharmaceutic Services") OR ("Community Pharmaceutic Service") OR ("Pharmaceutic Service Community") OR ("Pharmaceutic Services Community") OR ("Service Community Pharmaceutic") OR ("Community Pharmaceutical Services") OR ("Community Pharmaceutical Service")

OR ("Service Community Pharmacy") OR ("Pharmacy Service Clinical") OR ("Service Clinical Pharmacy") OR ("Clinical Pharmacy Services") OR ("Pharmacy Services Clinical") OR ("Services Clinical Pharmacy") OR ("Clinical Pharmacy Service") OR ("Pharmacy Residencies") OR ("Pharmacy Residency") OR ("Medication Management") OR ("medication reconciliation")
("Care Primary Health") OR ("Primary Care") OR ("Care Primary") OR ("Primary Healthcare") OR ("Healthcare Primary") OR ("Health Services Community") OR ("Community Health Service") OR ("Health Service Community") OR ("Service Community Health") OR ("Services Community Health") OR ("Community Health Care") OR ("Care Community Health") OR ("Health Care Community") OR ("Community Healthcare") OR ("Community Healthcares") OR ("Healthcare Community") OR ("Healthcares Community") OR ("Community Health Services") OR ("Preventative Medicine") OR ("Medicine Preventative") OR ("Preventive Medicine") OR ("Preventive Care") OR ("Care Preventive") OR ("Preventative Care") OR ("Care Preventative") OR ("Health Care Comprehensive") OR ("Comprehensive Healthcare") OR ("Healthcare Comprehensive") OR ("First Line Care") OR ("Health Care Primary") OR ("Community Care Service") OR ("Community Care Services") OR ("Preventive Health Care") OR ("Secondary Health Care") OR ("Secondary Healthcare") OR ("Care Secondary") OR ("Secondary Care") OR ("Secondary Cares") 


\section{Inclusion and exclusion criteria}

This systematic review provides the following inclusion and exclusion criteria for the studies found in the searches. Inclusion: studies with adult patients, older than 18 years; outpatient or hypertensive patients attending community pharmacies or primary care/ preventive health units; intervention carried out on the basis of pharmacotherapeutic monitoring; minimum of two consultations performed by the pharmacist in the intervention group; the pharmacist attending individually or inserted in a multi-professional team, but the intervention should not depend on the team, but exclusively on the pharmacist; assessment of blood pressure should be included. Exclusion: studies that consider the population: pregnant women; patients with cognitive impairment; with moderate to severe chronic kidney disease; not receiving pharmacological treatment for systemic arterial hypertension; without diagnosis of systemic arterial hypertension; narrative or integrative reviews, dissertations or theses; editorials; news; commentaries; letters to the editor; abstracts published in annals of scientific journals or congresses; guidelines; studies that develop pharmaceutical care without reviewing pharmacotherapy or pharmacotherapeutic monitoring or management of the health condition; studies that do not address pharmacotherapeutic follow-up in its aspects as a private service, in an individualized way, with the elaboration of a therapeutic plan, monitoring the results and with the systematic recording of patient data; studies without a comparator for outcomes.

\section{Eligibility}

Eligibility of the studies found will be analyzed for the studies included in the review and will follow the following criteria according to the previously prepared checklist (Table IV):

TABLE IV - Checklist for eligibility criteria

\begin{tabular}{|c|c|c|c|}
\hline Name of reviewer & Identification of the study & Eligibility criteria & Confirmation of eligibility \\
\hline $\begin{array}{l}\text { Who is making the search } \\
\text { and assessing the studies }\end{array}$ & $\begin{array}{ll}\text { - } & \text { Author } \\
\text { - } & \text { Title } \\
\text { - } & \text { Reference. }\end{array}$ & $\begin{array}{l}\text { Meeting all the items } \\
\text { described in the } \\
\text { inclusion criteria; } \\
\text { Absence of biases that } \\
\text { strongly compromise the } \\
\text { credibility of the study; } \\
\text { Availability of data } \\
\text { to be included in the } \\
\text { systematic review. }\end{array}$ & $\begin{array}{l}\text { Considering the study } \\
\text { design, the intervention, } \\
\text { and the population } \\
\text { involved, does the } \\
\text { study have potential } \\
\text { to be included? (Yes } \\
\text { or No or Not clear) }\end{array}$ \\
\hline
\end{tabular}

Types of studies to be included

Original articles will be considered, without limitation of language, and those studies that seek to answer the question of this systematic review and fit the inclusion and eligibility criteria. We emphasize that clinical trials will be analyzed in a separate group from the other studies, and divided into randomized and non-randomized trials. In addition, meta-analyzes and systematic reviews will not be included for the analysis.

\section{Risk evaluation of bias (quality)}

Initially, the bias analysis will be performed by the Cochrane risk bias classification guideline (Higgins, Green, 2011). The risk of bias will be evaluated for 
clinical trials and observational studies in order to assess the quality of the studies, for which the Downs and Black instrument will be used, which compiles a checklist of 28 items (allows to check the general quality of the study, internal and external validity, bias, confusion, and the power of analysis).

\section{Data synthesis and tabulation}

The responsible researchers will carry out, independently, the initial screening of the studies by reading the title. Subsequently they will independently review the abstracts and keywords of the articles obtained from the initial screening. The articles selected after this stage will be analyzed by reading the full text and will be included provided they meet the inclusion, exclusion, and eligibility criteria of this systematic review. Conflict in the selection of articles will be mutually resolved; if not agreed between the authors, the third researcher will make the final decision.

\section{Data synthesis strategy}

Following the Higgins and Green (2011) recommendations, the quantitative and qualitative data will be extracted from the studies included in the review using the data worksheet Excel $2010\left(\right.$ Microssoft $\left.^{\circledR}\right)$. This step was drawn according to eligible items by the authors, reasoned in the Cochrane handbook (Table V).

TABLE V - Checklist for Data extraction in this protocol and building the worksheet for databank

Items

\begin{tabular}{ll}
\hline & Year \\
& Reference \\
Basic data & Country \\
& Author \\
& Planned sample number
\end{tabular}

Method details

Participants

Clinical scenario

1. Study Design

3. Follow-up time

7. Blinding scheme:

Outpatient

\section{Description}

2. Number of pharmaceutical consultations

4. Number of pharmacists (for follow-up)

5. Randomization ("adequate", "inadequate" or "not reported" by the study)

6. Allocation concealment (reported or unreported)

- Researchers - "yes", "no" or "not reported"

- Participants - "yes", "no" or "not reported"

- Outcome assessors - "yes", "no" or "not reported"

Number of patients randomized by treatment branch or divided between comparison groups (exposed and not exposed)

Number of follow-up losses per group

Primary/preventive care unit (regarding the basic health unit, there should also be other professionals, if not it can be considered as a community pharmacy)

Community pharmacy (regarding the drugstore or basic pharmacy,

with only the pharmacist as a health professional)

Others 
TABLE V - Checklist for Data extraction in this protocol and building the worksheet for databank

Items

Description

1. Educational / Empowerment

Intervention (Characteristics

of monitoring)

2. Multi-professional support

3. Patient compliance was worked on

4. Pharmacotherapy management model (SOAP, PW, DADER, Proper or unspecified)

Take note of the brand label and company that made it

- Definition of each outcome investigated with its respective diagnostic criteria.

- Unit of measurement (if applicable) used as the gold standard (yes or no)

- For scales:

- report the upper and lower limits;

- report whether the score was high or low and whether it represented clinical improvement

Main outcomes

1. Blood pressure

2. Cardiovascular risk

Outcomes

3. Total Cholesterol

4. Fractions (LDL, VLDL, HDL)

5. Consumption of antihypertensive drugs ( $\mathrm{mg} /$ day or month or year)

6. Number of consultations (Basic, Cardiologist and Urgent/Emergency)

7. Number of examinations related to the follow-up of Systemic Arterial Hypertension

8. Percentage of patients with blood pressure at satisfactory levels (if this information is direct in the article, add which guideline was used as reference, if it is possible to calculate the percentage, the American guideline ACC / AHA should be used as reference)

- For each outcome, report categorical and/

Results or continuous and/or discrete variables.

- Subgroups to be analyzed: calculate the number of events to the total number of patients in each group

Sociodemographic descriptive data

- Average age

- Gender percentage

- Average income

- Schooling

- Ethnicity

- Previous history of diseases

- Comorbidities

- History of health events associated with systemic arterial hypertension 
TABLE V - Checklist for Data extraction in this protocol and building the worksheet for databank

Items

Description

\begin{tabular}{ll}
\hline & - Hypertension and cardiovascular risk staging \\
& - Oody Mass Index \\
Clinical parameters of interest for & Obesity \\
systemic arterial hypertension & Abdominal circumference \\
& - Smoker \\
& Drinker \\
\hline
\end{tabular}

Legend: Data on baseline characteristics of patients between the studies summarize clinical heterogeneity. The difference between the comparative basis of the study population may influence the extrapolation of the results. Thus, the subgroups or confounding factors for the clinical results achieved by pharmaceutical care that may contribute to the inconsistency of the findings will be predicted in the protocol of this review: difference in the groups among the variables of age, gender, comorbidities, for example. It should be emphasized that the intervention performed by pharmaceutical care must refer to clinical interventions and not merely educational interventions, empowerment and adherence to pharmacotherapy.

\section{Analysis of the results}

Before the time of consensus between the two researchers, the agreement between them will be analyzed by the Kappa coefficient, with a value above 0.80 being acceptable, otherwise there will be a need to restructure a new search strategy. The Mann-Whitney test will be performed to test the difference between the quality scores of the studies in which pharmaceutical care presents favourable and unfavourable clinical results. It is important pay attention to the analysis of subgroups or subsets: these will be planned after data extraction, which will allow to list important subgroups or sets to be analyzed.

\section{RESULTS}

In this paper we have worked on the strategy designed in this protocol to show the results achieved by the search strategies. It is important relate that the PRISMA flowchart is a gold standard to present the search results. It is important show search results found from all databases used and make clear the number of results after duplicates were removed; articles evaluated and excluded; full text with eligibility assessed and excluded after this because it did not contemplate the protocol; the final number of the studies included and how many from gray literature (Figure 2). 


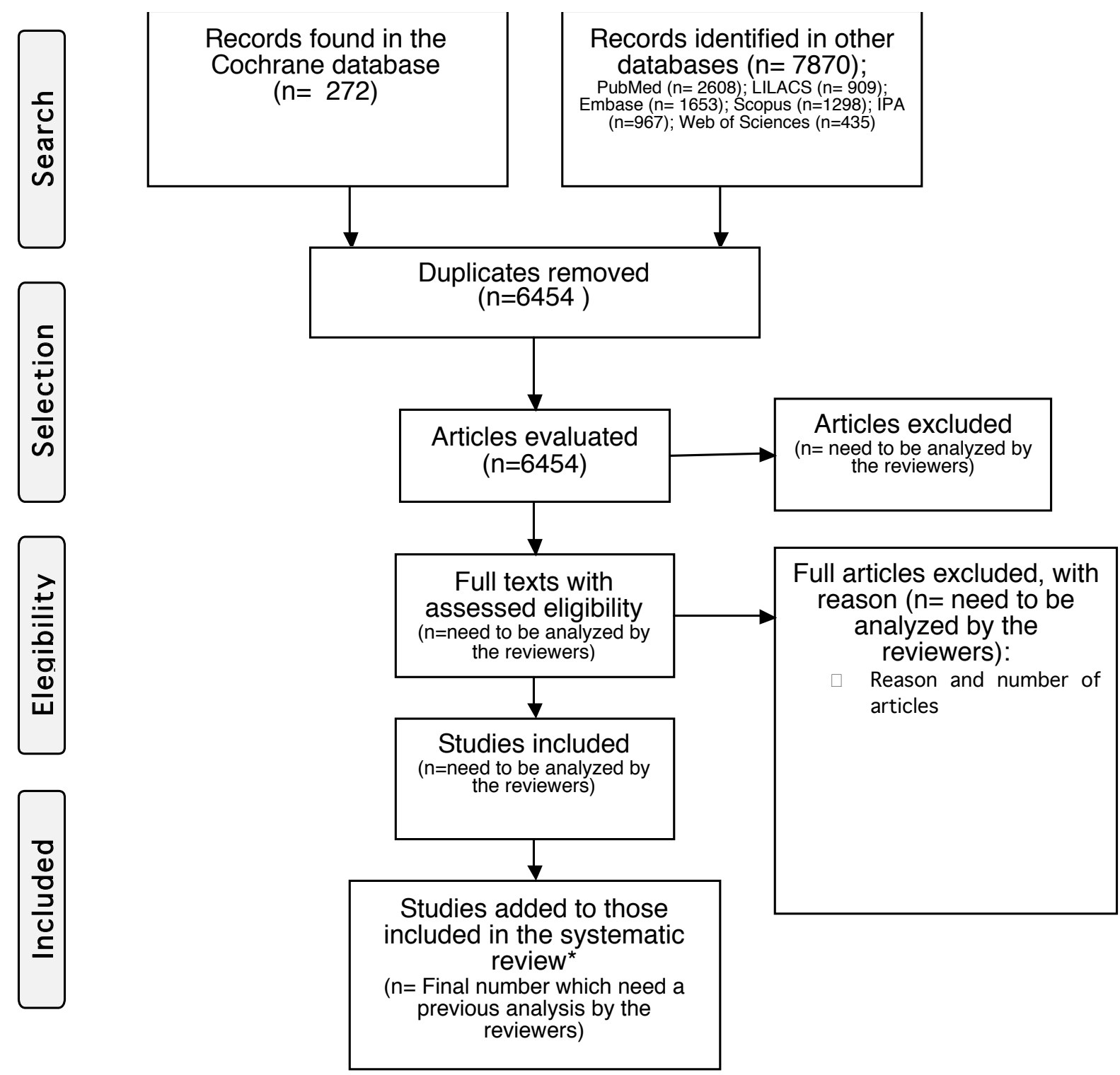

FIGURE 2 - Flowchart of how to present the search results, for instance, the search from this systematic review protocol.

\section{DISCUSSION}

Certainly the decision-making process is not a simple process. Furthermore, poorly made decisions can have serious consequences for the development of services, problem solving and efficiency of a system. One of the major problems for managers in the decisionmaking process is the distribution of their time to conduct rational decision-making processes (Bazerman, Moore, 2014).

The manager has an estimated $75 \%$ of their busy time with meetings, and with the dynamics of current relationships, the network, and the advent of the Internet, political relations established in meetings still strengthen this activity as one of the greatest time consumers of a manager (Laudon, Laudon, 2007). Thus it is extremely important to foster a fast and accurate decision-making process. In this context, systematic reviews are responsible for a large amount of evidence in decision-making. Consequently, robust systematic reviews may aid in the efficient decision-making process and be a ground for higher evidence levels regarding meta-analysis (Chiappelli et al, 2010).

In this scenario, we understand this paper's content is important to foster evidence synthesis. Additionally, this paper can influence the development 
of clinical pharmacy and pharmaceutical care and aid the government in the monitoring process regarding health policies in the public health system (Brazil, 2014; Brazil, 2018).

\section{CONCLUSION}

This paper presents a content to be extrapolated for other countries because care was taken to base it on general information for the construction of a systematic review, and was also based on the gold standard source for systematic reviews, the Cochrane handbook. Thus, the design of this paper can be replicated in several other systematic reviews and this fact tends to contribute to the improvement of the quality of the publications of studies worldwide.

\section{ACKNOWLEDGEMENTS}

We thank the São Paulo Research Foundation (FAPESP) funding agency for providing scholarship for the author Maurilio Souza Cazarim [grant number 2017/21240-0 from January 2018 to August 2018 and grant number 2016/03584-1 from August 2016 to August 2019], URL (http://bvs.fapesp.br/en/ pesquisador/673633/maurilio-de-souza-cazarim/), thus, it has contributed to the development of this paper. We highlight the funder had no role in study design, data collection and analysis, decision to publish, or preparation of the manuscript. Opinions, assumptions and conclusions or recommendations expressed in this article are the responsibility of the authors and do not necessarily reflect the viewpoint of FAPESP.

\section{CONFLICT OF INTEREST}

The authors confirm that the content of this paper has no conflict of interest.

\section{REFERENCES}

Aguwa CN, Ukwe CV, Ekwunife OI. Effect of pharmaceutical care programme on blood pressure and quality of life in a Nigerian pharmacy. Pharmacy World \& Science. 2008 Jan 1;30(1):107-10.

Araújo AL, Ueta JM, Freitas OD. Assistência farmacêutica como um modelo tecnológico em atenção primária à saúde. Rev. Ciênc. Farm. Básica Apl. 2005;26(2):87-92.
Bazerman, MH, Moore D. Processo Decisório. 8 ed. Rio de Janeiro: Elsevier, 2014.

Brazil. Ministério da Saúde. Secretaria de Ciência, Tecnologia e Insumos Estratégicos. Departamento de Assistência Farmacêutica e Insumos Estratégicos. Cuidado Farmacêutico na Atenção Básica. Caderno 1: Serviços Farmacêuticos na Atenção Básica. Brasília, DF, 2014. Available:<http://bvsms. saude.gov.br/bvs/publicacoes/servicos_farmaceuticos_ atencao_basica_saude.pdf $>$. Accessed: 17 July 2018.

Brazil. Ministry of Health. Institutional Development Support Program for Brazilian Public Health System (PROADISUS). [on line], Homepage. Ações e programas. PROADISUS. 2018. Available: http://portalms.saude.gov.br/acoes-eprogramas/proadi-sus. Accessed: 02 November 2018.

Brazil. Secretaria de Atenção à Saúde. Departamento de Atenção Básica. Documento de diretrizes para o cuidado das pessoas com doenças crônicas nas Redes de Atenção à Saúde e nas linhas de cuidado prioritárias. Brasília (DF): Ministério da Saúde, 2012. Available: http://bvsms.saude.gov.br/bvs/ publicacoes/diretrizes\%20_cuidado_pessoas\%20_doencas_ cronicas.pdf. Accessed: 02 November 2018.

Brazilian Society of Cardiology - BSC. VI Brazilian Guidelines on Hypertension. Arq Bras Cardiol. 2010;95(1 supl 1):1-51. doi: 10.1590/S0066-782X2004001000004

Castro MS, Correr CJ. Pharmaceutical care in community pharmacies: practice and research in Brazil. Annals of Pharmacotherapy. 2007 Sep;41(9):1486-93.

Cazarim MS, Freitas O, Penaforte TR, Achcar A, Pereira LRL. Impact assessment of pharmaceutical care in the management of hypertension and coronary risk factors after discharge. PloS One. 2016; https://doi.org/10.1371/journal. pone. 0155204 .

Chiappelli F, Brant XM, Neagos N, Oluwadara OO, Ramchandani MH. Evidence-based practice: toward optimizing clinical outcomes. Chiappelli $\mathrm{F}$, editor. $1^{\text {st }}$ ed. Berlin Heidelberg: Springer; 2010.

Cipolle RJ, Strand LM, Morley PC. Pharmaceutical care practice. 1st ed. New York: McGraw-Hill; 1998. ISBN 9780-07-175638-9.

Downs SH, Black N. The feasibility of creating a checklist for the assessment of the methodological quality both of randomised and non-randomised studies of health care interventions. Journal of Epidemiology \& Community Health. 1998 Jun 1;52(6):377-84.

Higgins JPT, Green S. Cochrane Handbook for Systematic Reviews of Interventions Version 5.1 [updated March 
2011]. The Cochrane Collaboration, 2011. Available: www. cochrane-handbook.org. Accessed: 18 July, 2018.

Laudon, KC, Laudon, JP. Sistemas de Informação Gerenciais. 7 ed. São Paulo: Pearson/Prentice Hall, 2007.

Lyra DP, Marcellini PS, Pelá IR. Effect of pharmaceutical care intervention on blood pressure of elderly outpatients with hypertension. Braz J Pharm Sci. 2008; https://doi. org/10.1590/S1516-93322008000300015.

Malachias MVB, Souza WKSB, Plavnik FL, Rodrigues CIS, Brandão AA, Neves MFT. Brazilian Society of Cardiology. VII Brazilian Guidelines on Hypertension. Arq Bras Cardiol. 2016; https://doi.org/10.5935/abc.20160140

Moher D, Liberati A, Tetzlaff J, Altman DG. Preferred reporting items for systematic reviews and meta-analyses: the PRISMA statement. Annals of internal medicine. 2009 Aug 18;151(4):264-269.

Organização Pan-Americana De Saúde (OPAS). Consenso brasileiro de Atenção Farmacêutica: proposta. Brasília, DF: Organização Pan-Americana da Saúde, 24 p. 2002.

Pereira LR, de Freitas O. A evolução da Atenção Farmacêutica e a perspectiva para o Brasil. Rev Bras Cien Farm. 2008 Dec 1;44(4):601-12.
Souza WA, Yugar-Toledo JC, Bergsten-Mendes G, Sabha M, Moreno H. Effect of pharmaceutical care on blood pressure control and health-related quality of life in patients with resistant hypertension. Am J Health-System Pharm. 2007 Sep 15;64(18):1955-61.

World Health Organization (WHO). Cardiovascular diseases (CVDs). Media centre: Fact sheets. 2017. Available: http:// www.who.int/mediacentre/factsheets/fs317/en/. Accessed: 02 June 2018.

World Health Organization (WHO). Global health observatory data repository. Cardiovascular diseases, deaths per 100.000 . 2014. [online]. Disponível em: <http://apps.who.int/gho/ data/node.main.A865CARDIOVASC ULAR?lang $=$ en $>$. Accessed: 06 June 2018.

World Health Organization (WHO). Media centre. Cardiovascular Diseases (CVDs). 2013. [online]. Disponível em: <http://www.who.int/mediacentre/factsheets/fs317/en/>. Accessed: 11 June 2018.

Zubioli A, Silva MA, Tasca RS, Curi R, Bazotte RB. Pharmaceutical consultation as a tool to improve health outcomes for patients with type 2 diabetes. Brazilian Journal of Pharmaceutical Sciences. 2013 Mar;49(1):85-94.

Received for publication on $30^{\text {th }}$ November 2018 Accepted for publication on $15^{\text {st }}$ April 2019 